\title{
SISTEMÁTICA, FILOGENIA Y BIOGEOGRAFÍA DE MYRMECOPHILA (ORCHIDACEAE)
}

\author{
Germán Carnevali ${ }^{1}$, José Luis Tapia ${ }^{1}$, Norris H. Williams ${ }^{2} \&$ W. Mark Whitten ${ }^{2}$ \\ ${ }^{1}$ Herbario CICY, Centro de Investigación Científica de Yucatan, México \\ ${ }^{2}$ Florida Museum of Natural History. Gainesville FL 32611-7800. U.S.A.
}

El género Myrmecophila Rolfe ha sido tradicionalmente incluido dentro de un concepto amplio de Schomburgkia Lindl. Sin embargo, recientes reconstrucciones filogenéticas basadas en secuencias de ADN indican que el grupo es basal dentro del complejo de taxa alrededor de Cattleya Lindl. s.l. y sólo lejanamente relacionado con las verdaderas Schomburgkia (las que están relacionadas con Laelia Lindl., un género restringido a elevaciones por encima de 1000 m en México central). Las "semejanzas" entre Schomburgkia s.s. y Myrmecophila (e.g., los miembros del perianto ondulados, las inflorescencias con largos pedúnculos y los ocho polinios) son el resultado de convergencia o de retención de caracteres plesiomórficos. La distinción entre ambos grupos ha sido reconocida desde hace mucho tiempo, pero a nivel seccional (e.g., Schlechter 1913, Foldats 1979). En 1917, Rolfe por primera vez sugiere tratar el grupo como un género aparte pero su propuesta no fue seguida por los autores posteriores (e.g., Williams 1946, Ames \& Correll 1953). Más recientemente, Kennedy (1979) hizo una propuesta convincente para la resurrección del género, que ha sido aceptada por la mayoría de los orquideólogos que trabajan en el área de distribución del grupo (e.g. Dressler 1993, McLeish et al. 1995, Espejo-Serna \& López-Ferrari 1997, Carnevali, Tapia-Muñoz \& Ramírez 2001, Carnevali et al. 2001).

Un análisis cladístico de todas las Laeliinae usando secuencias de ADN (van den Berg et al. 2000), identifica claramente a Myrmecophila como el grupo hermano del clado que incluye a los géneros del antiguamente llamado complejo Cattleya. En otras palabras, se trata de un grupo basal que hizo divergencia temprana de los ancestros que originaron a los géneros Brassavola R.Br., Rhyncholaelia Schltr., Cattleya, Sophronitis Lindl. y los miembros brasileños del género Laelia tal como se los ha circunscrito hasta hace poco. Por ello, no está relacionado con las verdaderas Laelias, tipificadas por Laelia grandiflora (Llave \& Lex. ) Lindl. [= Laelia speciosa (H.B.K.) Schltr.)] ni con Schomburgkia (tipificadas por Schomburgkia crispa Lindl.), grupos que no pertenecen a este clado de taxa alrededor de Cattleya.

Myrmecophila es fácilmente reconocible entre otras Laeliinae por sus plantas relativamente grandes con pseudobulbos homoblásticos (de varios entrenudos no diferenciados), huecos, portando (1-)2-3(-4) hojas en los entrenudos apicales y con inflorescencias largamente pedunculadas con flores vistosas que típicamente poseen sépalos y pétalos ondulados. Las plantas, usualmente epífitas (raramente litófitas o subterrestres), viven siempre asociadas con hormigas que forman colonias que habitan el interior de los pseudobulbos huecos. Se sabe que estas hormigas protegen tanto a la orquídea como a los forofitos de la herbivoría (Dejean et al. 1995).

Un estudio sistemático del género permite reconocer ocho especies y un híbrido natural. Las especies y sus distribuciones se presentan en la Tabla 1. Las especies se distribuyen naturalmente desde la costa caribe de Venezuela, donde crece una especie endémica, M. humboldtii, hasta el sureste de México donde se encuentra un centro de diversidad. Hay especies disyuntas en los estados mexicanos del Pacífico (Michoacán, Colima, Jalisco y Nayarit) donde crece Myrmecophila galeottiana y las vertientes pacíficas en Nicaragua, Honduras y El Salvador, donde crece la muy distintiva especie Myrmecophila wendlandii. Hay una especie disyunta en las Islas Caimán, M. thompsoniana. Por último, hemos reconocido un híbrido natural entre Myrmecophila christinae y M. brysiana, M. x lagu- 
Tabla 1. Especies de Myrmecophila reconocidas en este estudio y sus distribuciones. Abreviaciones. $\mathrm{B}=\mathrm{Belice} ; \mathrm{CR}=$ Costa Rica; ES = El Salvador; $\mathrm{G}=$ Guatemala; $\mathrm{H}=$ Honduras; $\mathrm{N}=$ Nicaragua; $\mathrm{M}=$ México; $\mathrm{g}=$ Costa del Golfo de México, $\mathrm{p}=$ costa del Pacífico.

\begin{tabular}{l|l}
\hline ESPECIES & DISTRIBUCIÓN \\
\hline M. brysiana (Lem.) G.C. Kenn. & CR, H, N, G, B, M (g) \\
M. christinae Carnevali \& Gómez-Juárez & $\mathrm{G}, \mathrm{B}, \mathrm{M}(\mathrm{g})$ \\
M. galeottiana (A. Rich.) Rolfe & $\mathrm{M}(\mathrm{p})$ \\
M. grandiflora Carnevali, Tapia-Muñoz \& I. Ramírez & $\mathrm{M}(\mathrm{g})$ \\
M. humboldtii (Rchb. f.) Rolfe & Venezuela \\
M. thompsoniana (Rchb. f.) Rolfe & Islas Caimán \\
M. tibicinis (Batem.) Rolfe & CR, H, N, G, B, M (g) \\
M. wendlandii (Rchb. f.) G.C. Kenn. & $\mathrm{N}, \mathrm{H}, \mathrm{G}, \mathrm{ES}$ \\
M. x lagunae-guerrerae Carnevali, Ibarra.González \& Tapia-Muñoz & $\mathrm{M}(\mathrm{g})$ \\
\hline
\end{tabular}

nae-guerrerae. Las especies del género son fácilmente reconocibles por combinaciones de caracteres florales y vegetativos y tienen distribuciones geográficas y ecológicas coherentes. Los patrones de coloración floral resultaron críticos en la delimitación específica, así como la morfología labelar y la estructura de la inflorescencia. Aún quedan problemas por ser esclarecidos en relación a cuántos taxa se encuentran dentro de la circunscripción actual de $M$. brysiana y si se deben reconocer taxa subespecíficos en las Myrmecophilas de las Islas Caimán (Dressler \& Carnevali 2000).

Una vez respondida la pregunta de cuántas especies constituyen el género y cuáles son sus límites, nos planteamos preguntas sobre las relaciones entre las especies. Por otra parte, la evolución de caracteres morfológicos selectos resulta de interés (número de entrenudos de los pseudobulbos, ramificación de la inflorescencias, textura floral, así como las distribuciones de los diversos grupos).

Para ello, seleccionamos dos taxa como grupos externos, Encyclia cordigera (H.B.K.) Dressler y Schomburgkia splendida Schltr. y como grupo interno incluimos todos los taxa descritos del género (con la excepción del híbrido natural). Los árboles obtenidos fueron enraizados con E. cordigera como grupo más externo.

Un total de 46 caracteres morfológicos, ocho caracteres de anatomía foliar y secuencias de ADN (ITS 1 y 2) fueron usados en la reconstrucción de las relaciones filogenéticas.
Se realizaron análisis separados de los caracteres morfológicos y de las secuencias de ADN. Luego, un análisis combinado fue llavado a cabo con el uso de los programas Winclada y Nona. Para la identificación de las topologías más parsimoniosas se utilizó el algoritmo del Ratchet con 200 iteraciones y 5 caracteres muestreados en cada iteración. Para evaluar el apoyo estadístico de los clados reconocidos, se realizó un analisis de Jacknife con 1000 réplicas. En todos los análisis, Myrmecophila resultó ser un grupo monofilético. Las topologías de los árboles de consenso de todos los análisis (morfología, ITS y combinado) resultaron consistentemente similares. Luego del reconocimiento y descarte de los caracteres no informativos filogenéticamente, el análisis combinado identificó un solo árbol más parsimonioso $(L=97$, $\mathrm{CI}=71, \mathrm{RI}=73$; Figura 1). Veinticuatro caracteres moleculares resultaron ser informativos, 10 de ellos sinapomórficos para el género Myrmecophila.

El único árbol obtenido (Figura 1) identifica a Myrmecophila humboldtii como el miembro más basal del género. Esta especie es el grupo hermano de dos clados, uno compuesto de dos especies (M. galeottiana y M. wendlandii), aquí llamado "Clado Pacífico" y el otro que incluye al resto del género, aquí llamado el "Clado Atlántico". Dentro del Clado Atlántico, la especie de las Islas Caimán es el taxón hermano del resto. Dentro de este Clado Atlántico, M. christinae y M. grandiflora son especies hermanas. Entre las sinapomorfias morfológicas de Myrmecophila destacan los pseudobulbos homoblásticos, huecos. 


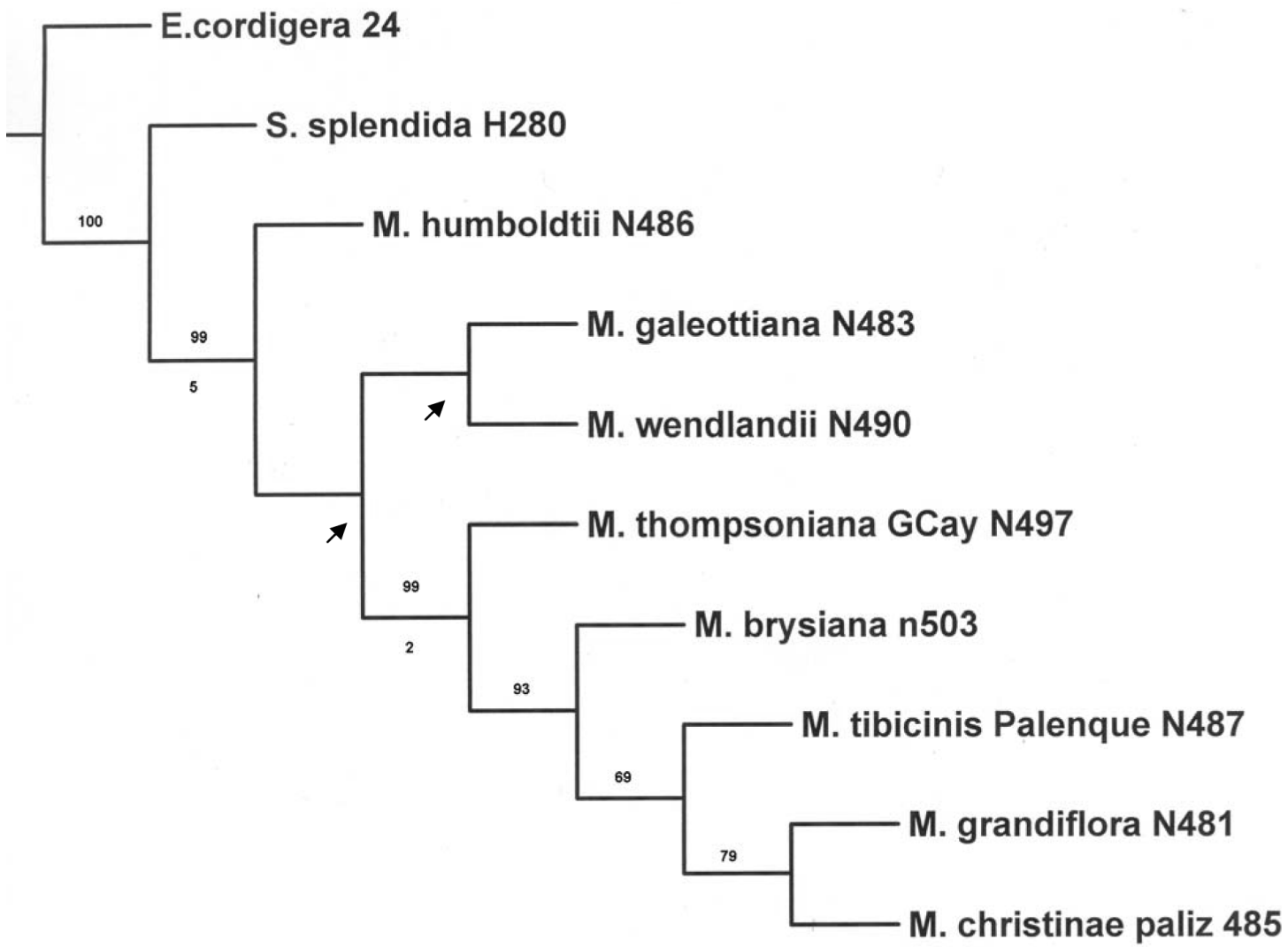

Figura 1. Único árbol más parsimonioso hallado en el análisis combinado (morfología, anatomía e ITS 1 \& 2). Números arriba de las ramas representan valores de Jacknife y los números por debajo indican los valores de apoyo en el análisis de Bremer. Nudos con flechas indican clados que colapsan en el árbol de Jacknife.

El Clado Pacífico tiene como sinapomorfias que lo apoyan la floración después de la estación lluviosa y la columna no ensanchada hacia el ápice. El Clado Atlántico está apoyado por las siguientes sinapomorfias: perianto glabro, nectario globular y pseudobulbos maduros de 5-7 entrenudos. Por otro lado, el clado formado por $M$. christinae (distribuida en Belice, Petén de Guatemala y Península de Yucatán) y M. grandiflora (México vertiente del Golfo en Oaxaca, Veracruz, Tamaulipas y Querétaro), un par de especies vicariantes, está apoyado por las inflorescencias usualmente simples, el labelo muy abierto en posición natural, las anteras mucho más anchas que largas y la posesión de un reborde conspicuo en el ápice de las anteras.

La topología del cladograma obtenido sugiere que el género pudo haber tenido una distribución más amplia que se extendía desde el norte de Sur
América, toda Centro América (ambas costas) y las Antillas y que hoy en día está representado por especies relictas en la región Pacífica, en las Antillas $\mathrm{y}$ en Venezuela. El género parece estar sufriendo una evolución activa en la Costa del Golfo de México y en el Caribe, donde crecen las cuatro especies más derivadas (el llamado complejo de Myrmecophila tibicinis), todas cercanamente relacionadas y con distribuciones vicariantes o especializaciones ecológicas cuando coexisten parapátricamente.

\section{Literatura Citada}

Ames, O. \& D.S. Correll. 1953. Laelia. In Orchids of Guatemala and Belize. Dover Publications, New York. p. 414-415.

Carnevali, G., J.L. Tapia-Muñoz, R. Jiménez-Machorro, L. Sánchez-Saldaña, L. Ibarra-González, I. M. Ramírez \& M P. Gómez-Juárez. 2001. Notes on the flora of the 
Yucatan Peninsula II: A synopsis of the orchid flora of the Mexican Yucatán Península and a tentative checklist of the Orchidaceae of the Yucatán Península Biotic Province. Harvard Pap. Bot. 5: 383-466.

Carnevali, G., J.L. Tapia-Muñoz, \& I. M. Ramírez, 2001. The status of Schomburgkia tibicinis var. grandiflora Lindl. (Orchidaceae) and a key to the Mexican species of Myrmecophila. Harvard Pap. Bot. 6: 245-251

Dejean, A., I. Olmsted \& R. R. Snelling. 1995. Tree-epiphyte-ant relationships in the low inundated forests of Sian Ka'an Biosphere Reserve, Quintana Roo, Mexico. Biotropica 27(1): 57-70

Dressler, R.L. 1993. Field Guide to the Orchids of Costa Rica and Panama. Cornell University Press, Ithaca \& Londres.

Dressler, R.L. \& G. Carnevali. 2000. Myrmecophila thompsoniana, the Wild Banana Orchid of the Cayman Islands. Orchid Digest 64(1): 25-30.

Espejo-Serna, A. \& A.R. López-Ferrari. 1997. Orchidaceae II. Part 8 of Las Monocotiledóneas Mexicanas: Una Sinopsis Florística. Consejo Nacional de la Flora de México, México, D. F.

Foldats, E. 1970. Schomburgkia Lindley. In Flora de Venezuela 15(3), Orchidaceae. Instituto Botánico, Caracas, Venezuela. pp. 455-468.

Kennedy, G.C. 1979. The genera Schomburgkia and Myrmecophila. Orch. Dig. 43(6): 205-211.

McLeish, I., N.R. Pearce \& B. R. Adams. 1995. Native Orchids of Belize. Balkema, Rotterdam, Netherlands.

Rolfe, R.A. 1917. Schomburgkia superbiens Lindl. Orch. Rev. 25: 49. 1917.

Schlechter, R. 1913. Die Gattung Schomburgkia Lindl. Orchis 7: 38--43.

van den Berg, C., W.E. Higgins, R.L. Dressler, W.M. Whitten, M.A. Soto Arenas, A. Culham \& M. W. Chase. 2000. A phylogenetic analysis of Laeliinae (Orchidaceae) based on sequence data from internal transcribed spacers (ITS) of nuclear ribosomal DNA. Lindleyana 15: 96-114.

Williams, L.O. 1946. The validity of the genus Schomburgkia. Darwiniana 5: 74-77.

Germán Carnevali obtuvo su licenciatura en biología en la Universidad Central de Venezuela; Maestría y Doctorado en la Universidad de Missouri-St. Louis, asociado con el Missouri Botanical Garden. Sus intereses son la sistemática y filogenia de varios grupos de Orchidaceae Neotropicales, principalmente los géneros Myrmecophila, Schomburgkia, Encyclia, Lophiaris, Cohniella y la subtribu Maxillariinae en general. Simultáneamente, tiene intereses en la florística de las Orchidaceae de la Península de Yucatán, América Central, las Guyanas, la Amazonía y Venezuela.

José Luis Tapia Muñoz obtuvo su licenciatura en la Universidad Veracruzana (Xalapa, Veracruz, México). Desde 1998 ha trabajado en el Herbario CICY del Centro de Investigación Científica de Yucatán, donde colabora con las investigaciones de Germán Carnevali. Sus intereses son la florística de la Península de Yucatán y la sistemática de varias familias, especialmente Convolvulaceae, Orchidaceae y Asteraceae. Trabajando con las Orchidaceae, sus intereses primordiales son el género Myrmecophila, el complejo Trichocentrum-Lophiaris-Cohniella y las Orchidaceae de la Provincia Biótica Península de Yucatán.

Norris H. Williams, Ph.D., es Curador de Plantas Vasculares en la Universidad de Florida, Herbario del Museo de Historia Natural de Florida, y ha trabajado en aromas florales, biología de la polinización, y en la sistemática y evolución de las orquídeas. En la actualidad dedica la mayoría de su tiempo al estudio de la sistemática molecular de las Orchidaceae neotropicales.

W. Mark Whitten, Ph.D., es Senior Biologist en el Herbario de la Universidad de Florida y ha trabajado con fragancias florales y biología de la polinización, así como en sistemática y evolución de las orquídeas. También Whitten está actualmente dedicando sus actividades de investigación al estudio de la sistemática molecular de las orquídeas del Neotrópico. 